\title{
Exploring Effects of Chitosan Oligosaccharides on Mice Gut Microbiota in in vitro Fermentation and Animal Model
}

\author{
Chen Zhang', Siming Jiao', Zhuo A. Wang ${ }^{*}$ and Yuguang Du* \\ State Key Laboratory of Biochemical Engineering, Institute of Process Engineering, Chinese Academy of Sciences, Beijing, \\ China
}

OPEN ACCESS

Edited by:

Maria Carmen Collado,

Instituto de Agroquímica y Tecnología

de Alimentos (IATA), Spain

Reviewed by:

F. Javier Moreno,

Instituto de Investigación en Ciencias

de la Alimentación (CIAL), Spain

Julio Galvez,

Universidad de Granada, Spain Habib ur Rehman,

University of Veterinary and Animal

Sciences, Pakistan

*Correspondence:

Zhuo A. Wang

wangzhuo@ipe.ac.cn

Yuguang Du

ygdu@ipe.ac.cn

${ }^{+}$Co-first authors

Specialty section:

This article was submitted to Microbial Symbioses,

a section of the journal

Frontiers in Microbiology

Received: 05 June 2018

Accepted: 18 September 2018

Published: 09 October 2018

Citation:

Zhang C, Jiao S, Wang ZA and

Du Y (2018) Exploring Effects

of Chitosan Oligosaccharides on Mice

Gut Microbiota in in vitro

Fermentation and Animal Model.

Front. Microbiol. 9:2388.

doi: 10.3389/fmicb.2018.02388
Chitosan oligosaccharides (COS) have shown positive effects on host gut health and influence on intestinal microbial community. However, the bioactivity and mechanism of COS on gut microbiota is still poorly understood. Here, we presented systematic studies of COS on mice fecal/gut microbiota. During in vitro fermentation of COS by mice gut microbiota, total bacterial population significantly decreased after 8-h COS treatment but was returned to the normal level after extended incubation. Consumption of COS and production of SCFAs suggested that COS were utilized by the microbe, although the consumption of chitosan pentasaccharides was obviously slower than others. COS treatments on mice fecal samples caused the decrease of potential pathogenic genera Escherichia/Shigella and the increase of genus Parabacteroides. In vivo animal study indicated that COS reduced population of probiotic genera Lactobacillus, Bifidobacterium and harmful genus Desulfovibrio, and increased abundance of genus Akkermansia. Phylum Proteobacteria was significantly inhibited by COS both in the animal model and in vitro fermentation. Our findings suggested that COS could reform the community structure of gut microbiota. The relationship among COS, gut microbiota and host health deserve further study.

Keywords: chitosan oligosaccharides, mice gut microbiota, in vitro fermentation, SCFAs, bacterial community

\section{INTRODUCTION}

Chitin the second most abundant biopolymer in the world. Chitosan oligosaccharides (COS), a mixture of oligomers of beta-1, 4-linked D-glucosamine, are prepared from chitosan, a $\mathrm{N}$-deacetylated derivative is of chitin (Thadathil and Velappan, 2014). The water-soluble and low toxicity of COS assists their versatile biological activities (Muanprasat and Chatsudthipong, 2017). COS have been reported to possess various properties like anti-microbial (Choi et al., 2001), antiinflammatory (Yousef et al., 2012), anti-diabetic activities (Lee et al., 2003), etc. Therefore, COS have good applicable values in food (Du et al., 2009) and pharmaceutical industries (Berger et al., 2004).

Gut microbiota can be considered as an extra organ with remarkable dynamics that influence the host gut health. Thousands of genes that encode carbohydrate-active enzymes (CAZymes) have been identified in the human gut microbiome (El Kaoutari et al., 2013). However, 
the human genome encodes, at most, only 17 enzymes for the digestion of food glycans, specifically starch, sucrose, and lactose and no polysaccharide lyases (Cantarel et al., 2012). COS as potential non-digestible oligosaccharides for the host, could be metabolized by gut microbiota. Dietary supplementation of COS has been found to improve gut barrier function, increase the population of Bifidobacterium spp. and Lactobacillus spp., and leave Escherichia coli counts unaffected in the cecum of weanling pigs (Yang et al., 2012). On the other hand, various studies reported that supplementation of COS significantly increased the abundance of Escherichia spp. in rats (Shang et al., 2017), but decreased the relative abundance of Lactobacillus in weanling pigs (Yu et al., 2017). Moreover, supplementation of COS and resistant starch mixtures alleviated metabolic disorders through synergistic actions, including positive manipulations on gut microbiota, lipid metabolism, and thickness of colonic mucosa layer in the rat (Shang et al., 2017).

Potential functions of oligosaccharides on gut microbiota could be tested using in vitro batch fermentation models that could exclude interferences from the host (Rastall, 2010; Payne et al., 2012). Limited number of studies had investigated the effect of COS on the gut microbiota in vitro and provided controversial conclusions. Lee et al. (2002) showed that COS stimulated the growth of Bifidobacterium bifudium and Lactobacillus spp. in pure cultures. On the contrary, Vernazza et al. (2005) did not observe an increase in Bifidobacteria counts in human fecal samples under COS treatments. This inconsistency through different researches might be due to different sources of COS and experimental settings (Lee et al., 2002; Mateos-Aparicio et al., 2016; Zou et al., 2016).

Based on these studies, the relationship among COS, gut microbiota and host gut health had been preliminarily established. However, the mechanism and causality of this relationship was still poorly explored. As an important part of the mechanism, the direct impact of COS on the gut microbiota needed to be further studied.

To investigate the effect of COS on the gut microbiota, we have determined the structure of microbiota in mice feces incubated with COS in in vitro batch cultures and colon contents in mice feeding with COS. To achieve this goal, COS with determined degree of polymerization (DP) and deacetylation (DD) was prepared. Batch cultures of mice feces were carried out with different concentrations of COS during in vitro anaerobic incubation. Consumption of COS as well as production of short chain fatty acids (SCFAs) and biogas were determined. Furthermore, the influence of COS upon both fecal microbiota in vitro and gut microbiota in animal was assessed. These studies are an important step toward a better understanding of the COS-microbe-host relationship.

\section{MATERIALS AND METHODS}

\section{Preparation and Identification of Chitosan Oligosaccharide}

Chitosan oligosaccharide with the deacetylation degree over 95\% and average molecular weight below $1 \mathrm{kDa}$ were prepared as previously described (Zhang et al., 1999). The content of COS was determined by UPLC-ESI-Q-TOF-MS (Waters Corp., Milford, MA, United States) equipped with an Acquity HSS T3 column $(100 \mathrm{~mm} \times 2.1 \mathrm{~mm}, 1.8 \mu \mathrm{m})$ (Supplementary Figure S1), showing a range of DP from 2 to 6 . COS with different DP from 2 to 6 were shown as COS-2, COS-3, COS-4, COS-5, and COS-6, respectively. Aqueous solutions $(5 \mu \mathrm{L})$ of COS $(10 \mathrm{mg}$ $\mathrm{mL}^{-1}$ ) were injected into HPLC for analysis. Mobile phase was composed of solution A (10 mM ammonium formate in water) and solution $\mathrm{B}$ (acetonitrile) with a gradient elution as follows: from 0 to $2 \mathrm{~min}, 15 \%$ (A) $85 \%$ (B); from 2 to $32 \mathrm{~min}, 15-50 \%$ (A) $85-50 \%$ (B); and from 32 to $33 \mathrm{~min}, 50-80 \%$ (A) $50-20 \%$

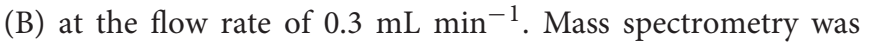
applied for the qualitative analysis under negative ion modes. The MS parameters were set up as follows: capillary voltage, $3 \mathrm{kV}$; cone voltage, $30 \mathrm{~V}$; source temperature, $120^{\circ} \mathrm{C}$; desolvation temperature, $550^{\circ} \mathrm{C}$; gas flows of cone and desolvation, 50 and $1000 \mathrm{~L} \mathrm{~h}^{-1}$. Mass spectrometry data were processed using Masslynx 4.1 software. COS samples were also analyzed using Acchrom S6000 HPLC system (Acchrom, China) equipment with an Acchrom XAmide column $(4.6 \mathrm{~mm} \times 250 \mathrm{~mm} \times 5 \mu \mathrm{m}$, Acchrom, China) coupled with the evaporation light-scattering detector. Mobile phase was composed of acetonitrile (A) and $100 \mathrm{mM}$ ammonium formate (B) with a gradient elution as follows: from 0 to $25 \mathrm{~min}, 90 \%$ (A) $10 \%$ (B); then from 26 to $70 \mathrm{~min}, 70 \%$ (A) $30 \%$ (B). The flow rate was $0.5 \mathrm{~mL} \mathrm{~min}^{-1}$ and the column temperature was $30^{\circ} \mathrm{C}$. The relative abundance of COS oligomers was evaluated by determining the peak area of each oligosaccharide component using COS oligomer standards as external standard (Dalian Glycobio Co., Ltd.). Each sample was analyzed for three times.

\section{Anaerobic Incubation}

Fresh feces (0.25 g) of male C57BL/6J mice were uniformly dispersed by vortex to get seed liquid in $50 \mathrm{~mL}$ a rich gut microbiota medium (GMM), which was prepared as described earlier (Goodman et al., 2011; Zhang et al., 2014). Anaerobic incubations were prepared by adding $3 \mathrm{~mL}$ of seed liquid into $27 \mathrm{~mL}$ of fresh GMM in 100-mL glass bottles. The stock solution of COS $\left(100 \mathrm{~g} \mathrm{~L}^{-1}\right)$ was sterilized by filtration, then added into samples to final concentrations of $0.1,1$, and $3 \mathrm{~g} \mathrm{~L}^{-1}$, respectively. Same volume of GMM was added to the control treatment. Bottles were sealed with butyl stoppers, then flushed with $\mathrm{N}_{2}$ for $3 \mathrm{~min}$ and incubated at $37^{\circ} \mathrm{C}$. After $72 \mathrm{~h}$ incubation, $3 \mathrm{~mL}$ cultured samples of the primary culture (G1) were transferred into the $27 \mathrm{~mL}$ GMM to get the second subculture (G2). The 3rd subculture (G3) was conducted after $168 \mathrm{~h}$ incubation of the G2. All tests were carried out in triplicate for each treatment.

\section{Gas and Short Chain Fatty Acids (SCFAs) Analysis}

Gaseous samples $(100 \mu \mathrm{L})$ were collected from the headspace of the bottle and injected into GC with a pressure-lock precision analytical syringe (Baton Rouge, LA, United States) and concentrations of $\mathrm{H}_{2}, \mathrm{CH}_{4}$, and $\mathrm{CO}_{2}$ were analyzed using a gas chromatograph GC-7890A (Agilent Technologies, 
United States), equipped with thermal conductivity detector (TCD), flame ionization detector (FID) and Electrical Conductivity Detector (ECD) (Zhang et al., 2014). Fermentation samples were centrifuged at $14,000 \times g$ for $10 \mathrm{~min}$ and the supernatants were filtered through $0.22 \mu \mathrm{m}$ (pore size) filters (Sangon, Shanghai, China) and stored at $-20^{\circ} \mathrm{C}$. The sediments were stored at $-80^{\circ} \mathrm{C}$ for DNA extraction. SCFAs including formate, acetate, butyrate and L-lactate were analyzed using an Acchrom S6000 high performance liquid chromatography (HPLC) system (Acchrom, China) equipment with a C18 column $(4.6 \mathrm{~mm} \times 250 \mathrm{~mm} \times 5 \mu \mathrm{m}$, Acchrom, China $)$ at $210 \mathrm{~nm}$ of UV absorbance. Five microliter sample was injected into the HPLC system for each run. Mobile phase was composed of $1 \%$ acetonitrile and $99 \% \mathrm{NaH}_{2} \mathrm{PO}_{4}$ with isocratic elution. The $\mathrm{pH}$ of mobile phase was adjusted to 2.0 with phosphoric acid.

\section{Nucleic Acid Extraction and Quantitative Analysis of Bacteria}

Cultured samples were taken from incubations at indicated time points. Microbial DNA was extracted following the protocol of FastDNA SPIN Kit with bead-beating using FastPrep-24 (MP Biomedicals, CA, United States). The copy number of bacterial 16S rRNA gene was determined using quantitative PCR in a StepOne real-time PCR system (Applied Biosystems) with the primer pair Ba519f (CAGCMGCCGCGGTAANWC) and Ba907r (CCGTCAATTCMTTTRAGTTT) (Lueders et al., 2004). Standards with a 10 -fold dilution series from $8.07 \times 10^{8}$ to $8.07 \times 10^{3}$ copies $\mu \mathrm{L}^{-1}$, were prepared from the purified plasmid DNA carrying bacterial 16S rRNA gene of Allobaculum stercoricanis (DSM 13633T).

\section{Animal Feeding Trial}

Male C57BL/6J (6 weeks old) mice were purchased from Model Animal Research Center of Nanjing University (Nanjing, China). After acclimation for 1 week, 10 mice were randomly divided into two groups $(n=5)$ : chow diet $(\mathrm{CD})$ group and CD + COS $(1 \mathrm{~g}$ $\mathrm{L}^{-1}$ in drinking water, about $200 \mathrm{mg} \mathrm{kg} \cdot \mathrm{d}^{-1}$ ) group. The standard CD diet (Aoke Xieli Co., Ltd., China) contained 20\% protein, $70 \%$ carbohydrate, and 10\% fat (Supplementary Table S1). After feeding for 5 months at $22^{\circ} \mathrm{C}$ with a $12-12 \mathrm{~h}$ dark-light cycle, all mice were euthanized and colon contents were sampled. Samples were immediately frozen in liquid nitrogen and stored at $-80^{\circ} \mathrm{C}$ until DNA extraction. The animal experiments were approved by the Animal Ethical Experimentation Committee of Chinese Academy of Sciences (permission number: SYXK2015-0002).

\section{Sequencing and Bacterial Community Analyses}

The V3-V4 region of bacterial 16S rRNA genes was subjected to PCR amplification. Purified amplicons were sequenced on the Illumina MiSeq platform by Allwegene Technology Inc. (Beijing, China). Raw sequences were analyzed by QIIME software package as described previously (Caporaso et al., 2010). Unique sequences were clustered into operational taxonomic units (OTUs) defined by 97\% similarity. Alpha and beta diversity were examined as well as redundancy analysis (RDA) and principal coordinate analysis (PCoA). Linear discriminant analysis effect size (LEfSe) was used to estimate taxonomic abundance and characterize differences between groups (Segata et al., 2011). All 16S rRNA pyrosequencing datasets had been deposited in GenBank Sequence Read Archive (SRA) database (Accession number: SRP114727 and SRP142222).

\section{Statistical Analysis}

All statistical analyses were carried out using GraphPad Prism (version 6, GraphPad Software Inc., San Diego, CA, United States). Data were presented as means \pm SD. Data of the $\mathrm{H}_{2}$ and SCFAs concentrations and bacterial population abundance were subjected to the parametric ANOVA analysis, along with the Tukey-Kramer test, to determine the significant differences between the treatments. LEfSe analysis of the treatment groups was performed on the basis of the results of the Kruskal-Wallis and Wilcoxon tests and the threshold on the logarithmic linear discriminant analysis (LDA) score was 2.0. The $P$-value $<0.05$ was regarded as statistical significance.

\section{RESULTS}

\section{In vitro Fermentation of COS in Mice Fecal Samples}

In the first $33 \mathrm{~h}, 0.76 \mathrm{~g} \mathrm{~L}^{-1}$ and $1.82 \mathrm{~g} \mathrm{~L}^{-1} \mathrm{COS}$ were degraded in samples treated with 1 and $3 \mathrm{~g} \mathrm{~L}^{-1} \mathrm{COS}$, respectively. Then, the degradation was slow down with only $0.15 \mathrm{~g} \mathrm{~L}^{-1}$ and $0.48 \mathrm{~g}$ $\mathrm{L}^{-1}$ COS were utilized in the following time (Figure 1A). The change of COS was not recorded in samples treated with $0.1 \mathrm{~g}$ $\mathrm{L}^{-1}$ COS since the concentration was below the detection limit (about $0.1 \mathrm{~g} \mathrm{~L}^{-1}$ ) of the HPLC instrument used in the experiment. To determine the degradation of different COS components by mice fecal microbiota, changes in the relative abundance of each COS oligomer were characterized through fermentation. Contents of major components of the original COS sample were COS-2 (DP2) 3.4\%, COS-3 (DP3) 17.8\%, COS-4 (DP4) $32.1 \%$, COS-5 (DP5) 25.9\%, and COS-6 (DP6) 12\%, respectively, accounted for $91.2 \%$ of total COS (Supplementary Figures S1, S2). During fermentation, concentrations of COS-2, 3, 4, and 6 all dropped quickly to undetectable level $\left(<0.1 \mathrm{~g} \mathrm{~L}^{-1} \mathrm{COS}\right)$ after $48 \mathrm{~h}$ (Figures 1B-D,F). Interestingly, degradation of COS-5 was obviously slower than other components (Figure 1E). Till the end of the incubation, almost 46 and $72 \%$ of COS- 5 still remained in the treatments with 1 and $3 \mathrm{~g} \mathrm{~L}^{-1} \mathrm{COS}$, respectively.

\section{$\mathrm{H}_{2}$, Lactate and SCFAs Dynamics}

$\mathrm{H}_{2}$, lactate and SCFAs were produced during the fermentation of COS in mice fecal samples (Figure 2). $\mathrm{H}_{2}$, acetate and butyrate showed a continuous accumulation in the samples with COS treatment (Figures 2A,C,E). The concentration of $\mathrm{H}_{2}$, acetate and butyrate was significantly higher in treatments with 1 and $3 \mathrm{~g} \mathrm{~L}^{-1}$ COS than that in 0 and $0.1 \mathrm{~g} \mathrm{~L}^{-1}$ COS treatments at $72 \mathrm{~h}$, respectively (Supplementary Figures S3A,C,E). At the 
A

COS-total

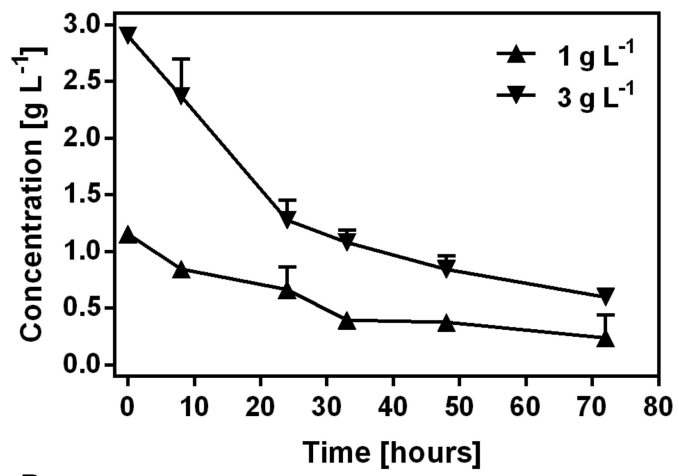

B

COS-2

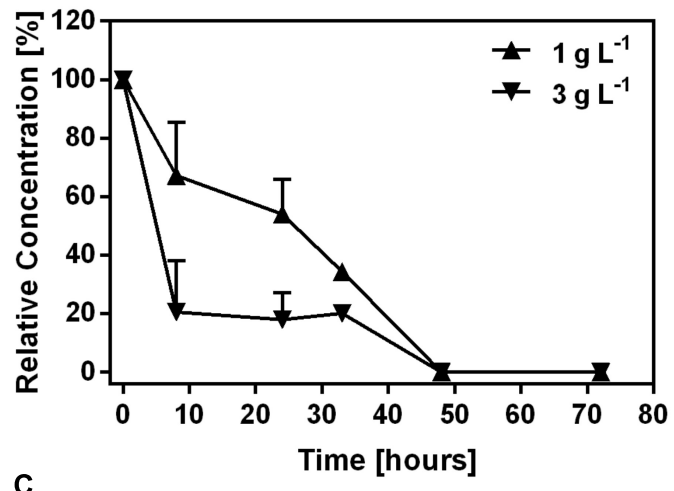

Cos-3

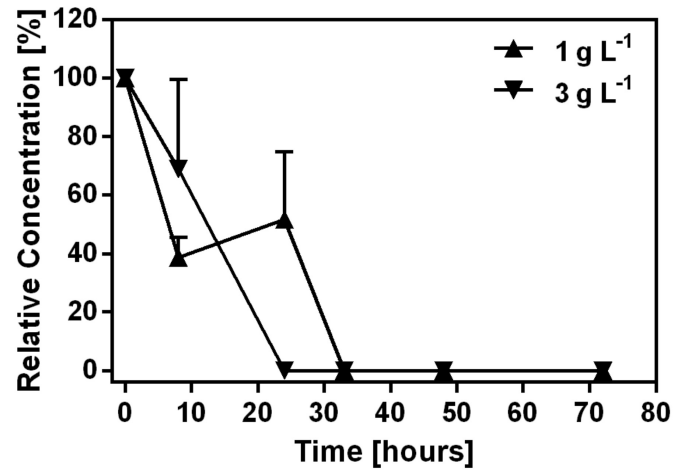

D

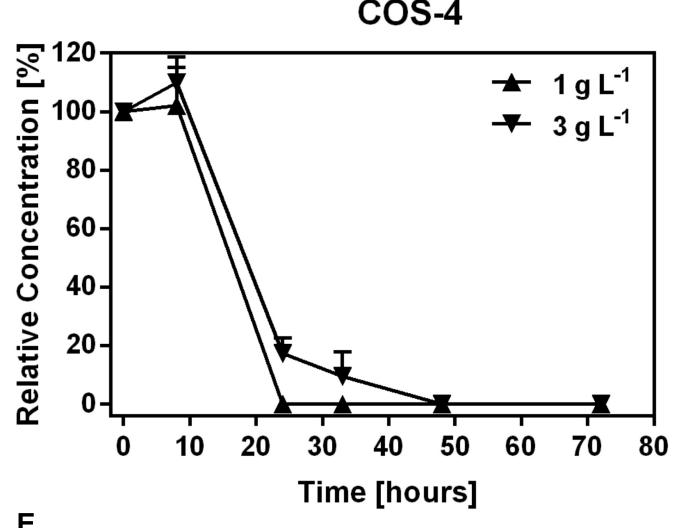

E

COS-5

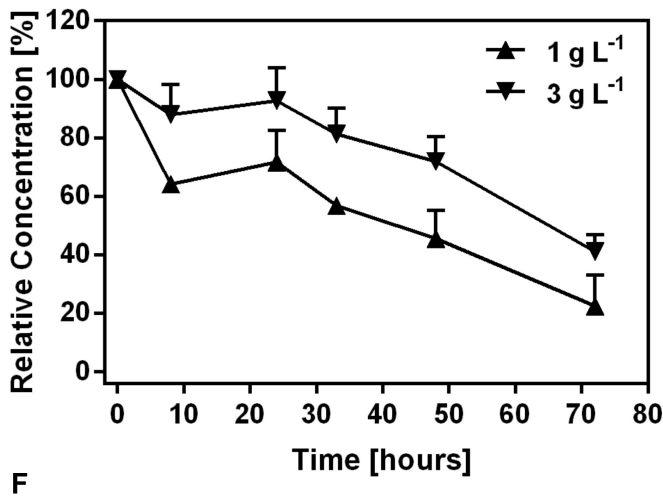

COS-6

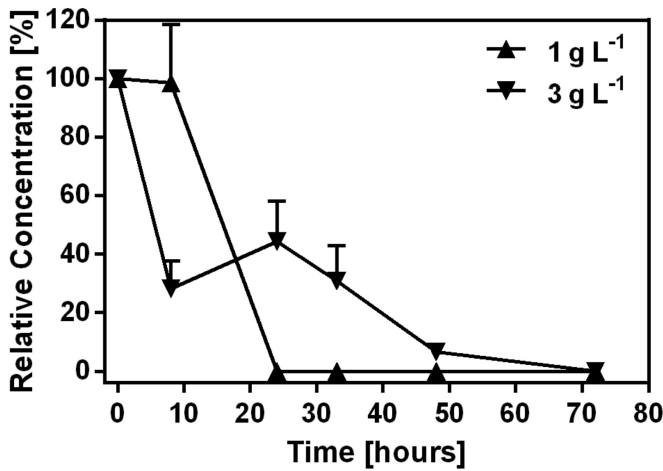

FIGURE 1 | Dynamics of COS polymers during COS treatment on mice feces. The concentration of COS-total (A), and the level of COS-2 (B), COS-3 (C), COS-4 (D), COS-5 (E), and COS-6 (F) in mice feces treated with 0.1, 1, and $3 \mathrm{~g} \mathrm{~L}^{-1}$ COS, respectively. COS with different degree of polymerization (DP) from 2 to 6 were shown as COS-2, COS-3, COS-4, COS-5, and COS-6, respectively. Data were collected at 0, 8, 24, 32, 48, and 72 h of the treatment. Data are represented as the means $\pm \operatorname{SD}(n=3)$.

end of incubation, the partial pressure of $\mathrm{H}_{2}$ was higher in COS treated samples $(3.1-3.9 \mathrm{kPa})$ than that in the control $(2.8 \mathrm{kPa})$. Acetate was the most abundant SCFAs and reached 36, 47.6, and $51.3 \mathrm{mM}$ during the incubation in treatments with $0.1,1$, and $3 \mathrm{~g} \mathrm{~L}^{-1}$ COS, respectively. In the control, acetate increased to $36.8 \mathrm{mM}$ at $48 \mathrm{~h}$, and then decreased to $31.7 \mathrm{mM}$ at the end of incubation (Figure 2C). Formate accumulated during the first $24 \mathrm{~h}$, then decreased to about $6.5 \mathrm{mM}$ after $48 \mathrm{~h}$ in all tests. The highest concentrations of formate were 15.4 and $18.4 \mathrm{mM}$ in fecal samples with 1 and $3 \mathrm{~g} \mathrm{~L}^{-1} \mathrm{COS}$, respectively. However, the accumulation of formate only reached a relative low level in the control and $0.1 \mathrm{~g} \mathrm{~L}^{-1} \mathrm{COS}$ treated groups (Figure 2B and Supplementary Figure S3B). In addition, the production of propionate could not be detected during the incubation.

In all tested groups, lactate accumulated during the first $24 \mathrm{~h}$, then dropped till the end of the incubation (Figure 2D). The highest concentration of lactate was above $32 \mathrm{mM}$ in the control and samples treated with $0.1 \mathrm{~g} \mathrm{~L}^{-1}$ COS, but only 
A

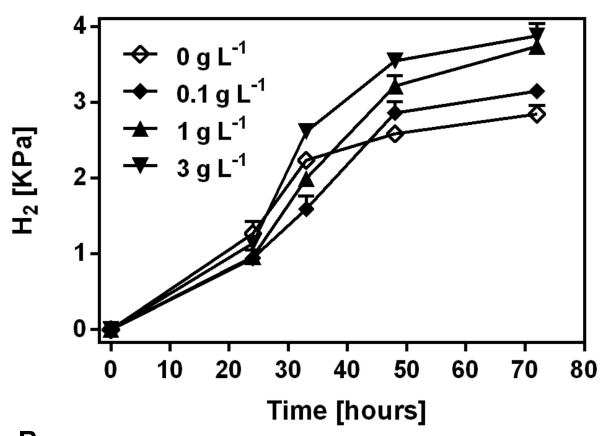

B

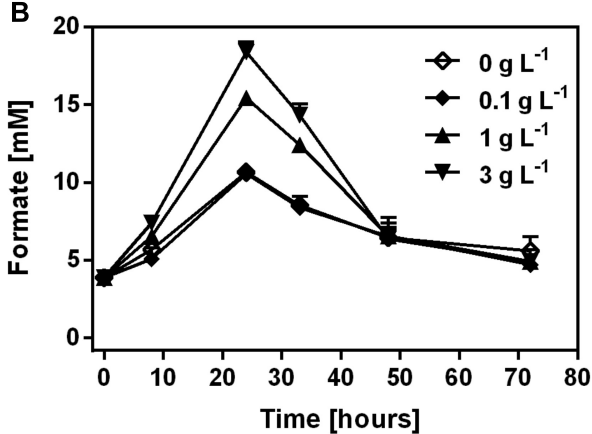

C

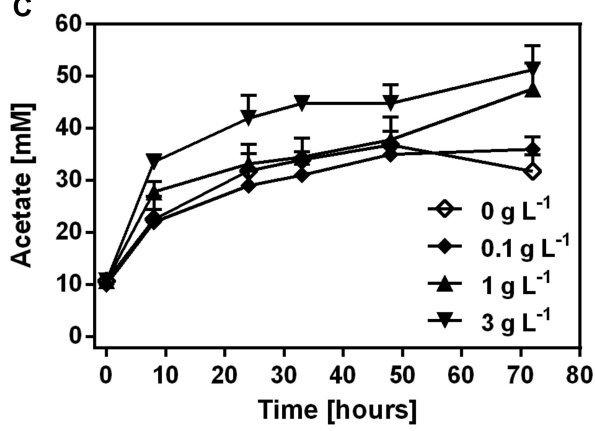

D

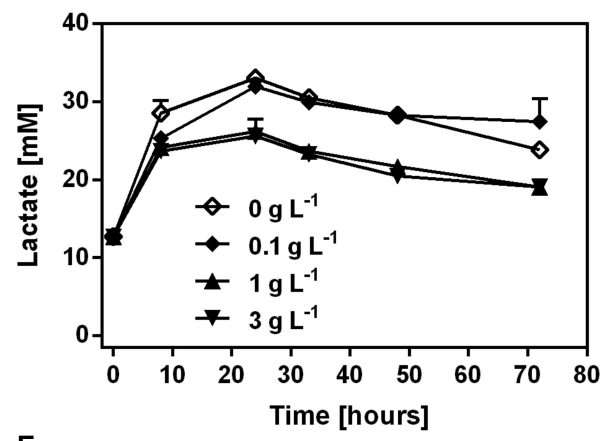

E

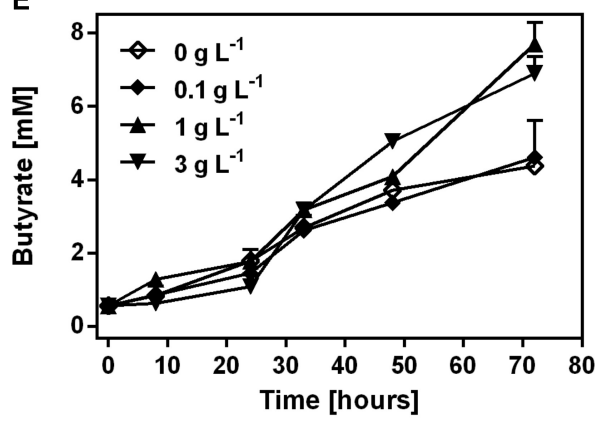

FIGURE 2 | Production of $\mathrm{H}_{2}$ and SCFAs by the mice fecal microbe during COS treatment. The concentration of $\mathrm{H}_{2}$ (A), formate (B), acetate (C), lactate (D), and butyrate (E) was analyzed in mice feces treated with $0,0.1,1$, or $3 \mathrm{~g} \mathrm{~L}^{-1} \mathrm{COS}$, respectively. Data were collected at $0,8,24,32,48$, and $72 \mathrm{~h}$ of the treatment. Data are represented as the means $\pm \mathrm{SD}(n=3)$.

$26 \mathrm{mM}$ in 1 and $3 \mathrm{~g} \mathrm{~L}^{-1} \mathrm{COS}$ treated groups. Thus, the lactate production was significantly inhibited by COS in relative high concentrations (Supplementary Figure S3D). It suggested that lactate-producing microbe in the fecal sample was inhibited by COS.

\section{Qualification of Bacterial Community}

The total bacterial 16S rRNA gene copy numbers were estimated by quantitative PCR (Table 1). After $8 \mathrm{~h}$ incubation, the copy numbers of bacterial 16S rRNA gene significantly decreased from $3.5 \times 10^{11}$ copies $\mathrm{mL}^{-1}$ to $1.1 \times 10^{9}$ copies $\mathrm{mL}^{-1}$ and $2.9 \times 10^{8}$ copies $\mathrm{mL}^{-1}$ in the $1 \mathrm{~g} \mathrm{~L}^{-1} \mathrm{COS}$ and $3 \mathrm{~g} \mathrm{~L}^{-1} \mathrm{COS}$ treated samples, respectively. However, copy numbers of bacterial $16 \mathrm{~S}$ rRNA gene under these treatments returned to the same level as the control group after $72 \mathrm{~h}$ incubation. Besides, the bacterial copy numbers were barely affected by the treatment with $0.1 \mathrm{~g} \mathrm{~L}^{-1}$ COS. Thus, the inhibitory effect of COS on the fecal bacteria was dependent on the concentration of COS as expected. However, the population of the bacterial community quickly recovered with the extended incubation time likely due to the degradation of COS as shown above.

\section{Change of Bacterial Community During in vitro Fermentation of Mice Feces With cos}

To determine the composition of the bacterial community, bacterial 16S rRNA gene pyrosequencing was performed. Alfadiversity of bacterial community of samples showed that, as the sequencing depth increased, the number of observed species also increased (Supplementary Figure S4A). There was no significant difference in richness (as indicated by rarefaction of observed species) and diversity between the control and samples treated with $0.1 \mathrm{~g} \mathrm{~L}^{-1} \mathrm{COS}$, while addition of $>1 \mathrm{~g} \mathrm{~L}^{-1} \mathrm{COS}$ 

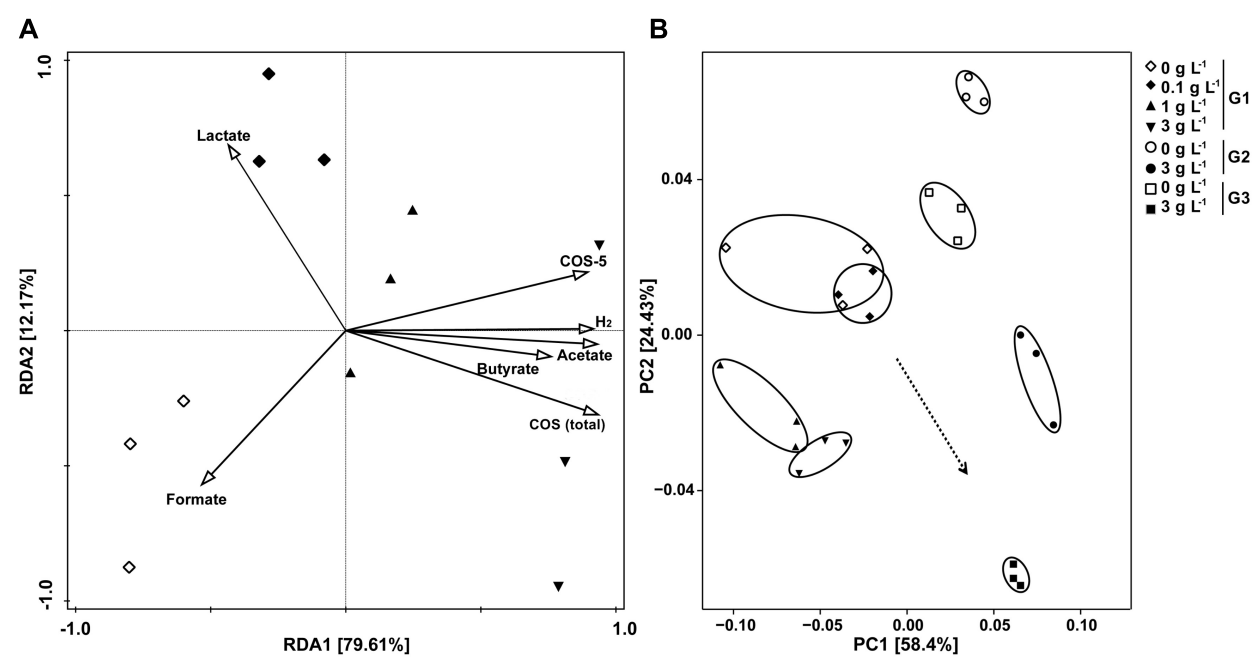

FIGURE 3 | Redundancy analysis (RDA) and UniFrac-based PCoA analysis on bacterial diversity influenced by COS treatment. RDA for samples from primary culture (G1) with COS (total), COS-5, $\mathrm{H}_{2}$, SCFA and acetate as environmental variables (A). The length of each arrow indicated the contribution of the corresponding parameter to the structural variation. UniFrac-based PCoA using the weighted version for samples from the primary culture and subculture (B). Mice stool incubated with $0,0.1,1$, or $3 \mathrm{~g} \mathrm{~L}^{-1} \mathrm{COS}$ at $72 \mathrm{~h}$ of the primary culture (G1), and 0 or $3 \mathrm{~g} \mathrm{~L}^{-1} \mathrm{COS}$ at the end of the 2nd subculture (G2), and 0 or $3 \mathrm{~g} \mathrm{~L}-1 \mathrm{COS}$ at the end of the 3rd subculture (G3).

significantly reduced both the richness and diversity of the bacterial community (Supplementary Figure S4B). RDA was conducted to examine the relationships between environmental properties and bacterial population distribution of the samples (Figure 3A). Content change of COS, COS-5, $\mathrm{H}_{2}$, acetate and butyrate explained $79.6 \%$ of the total variance of the bacterial population, while lactate and formate were related to $12.2 \%$ of the total variance. The concentration of COS was positively associated with the $\mathrm{H}_{2}$ partial pressure and the concentration of acetate and butyrate. However, there was a negative correlation between COS and lactate concentration. UniFrac-based principal coordinates analysis (PCoA) revealed a distinct clustering of bacteria composition for each experimental group (Figure 3B). Those results indicated that the addition of more than $1 \mathrm{~g} \mathrm{~L}^{-1} \mathrm{COS}$ markedly affected bacterial population distribution.

Phylogenetic analysis indicated that all tested samples comprised mainly phylum Proteobacteria, Firmicutes, Bacteroidetes, and Actinobacteria (Supplementary Figure S5). The abundance of the phylum Bacteroidetes, which was dominant with genus Parabacteroides, was the significantly increased $(P<0.05)$, while phylum Proteobacteria was remarkably decreased $(P<0.05)$ under $3 \mathrm{~g} \mathrm{~L}^{-1}$ COS treatment at the end of the 3rd subculture. The influence of COS on the relative abundance of bacterial community at the genus level was analyzed (Figure 4). The bacterial community structure in the $0.1 \mathrm{~g} \mathrm{~L}^{-1}$ COS-treated group remained similar to the control after $72 \mathrm{~h}$ of incubation. Enterobacteriaceae, accounting for about $30 \%$ of total bacterial abundance, predominated in both groups. Enterococcus accounted for 16-22\% of total bacterial abundance. Enterococcus was further enriched in samples treated with more than $1 \mathrm{~g} \mathrm{~L}^{-1}$ COS and accounted for 33-35\% of total bacterial population. In addition, Lactobacillus, Escherichia/Shigella decreased in abundance from $4.17 \%$ to $0.02-0.69 \%$ after COS treatments $\left(>1 \mathrm{~g} \mathrm{~L}^{-1}\right)$. The decreased abundance of Lactobacillus indicated the inhibitory effect of COS $\left(>1 \mathrm{~g} \mathrm{~L}^{-1}\right)$ on the growth of genus Lactobacillus, consistent with the experimental result of COS treatment on the growth of three different Lactobacillus strains in pure cultures (Supplementary Figure S8).

The trend of changed proportions of certain bacterial groups in the mice fecal sample under COS treatment was further intensified by subcultures (Figures 4B,C). The relative abundance of Proteus, Escherichia/Shigella, and Bacteroides decreased 2-5-fold in $3 \mathrm{~g} \mathrm{~L}^{-1}$ COS treated group. Furthermore, COS significantly stimulated the growth of Parabacteroides through transfer of subcultures. The relative abundance of Parabacteroides was 10 times higher in $3 \mathrm{~g} \mathrm{~L}^{-1} \mathrm{COS}$ treated group than that in the control.

Linear discriminant analysis (LDA) effect size (LEfSe) was performed to identified bacteria strains which exhibited obvious changes in relative abundance under COS treatment (Supplementary Figures S6, S7). Six different taxa were identified in samples treated with $1 \mathrm{~g} \mathrm{~L}^{-1}$ COS and 10 taxa in samples with $3 \mathrm{~g} \mathrm{~L}^{-1}$ COS (Supplementary Figures S6A,B). COS treatments significantly increased proportions of Enterococcus and Lactobacillales (Supplementary Figures S6E,F). On the contrary, the abundance of Enterorhabdus and Ruminiclostridium_9 significantly dropped under COS treatments. It is important to note that the relative abundance of Lactobacillus decreased significantly from 2 to $0.02 \%$ under $3 \mathrm{~g}$ $\mathrm{L}^{-1}$ COS treatment.

\section{In vivo Feeding Trial}

The gut microbiota was dominated by four major phyla, Firmicutes, Actinobacteria, Bacteroidetes, and Proteobacteria in the control mice (Figure 5A). With the supplementation of 


\section{A}

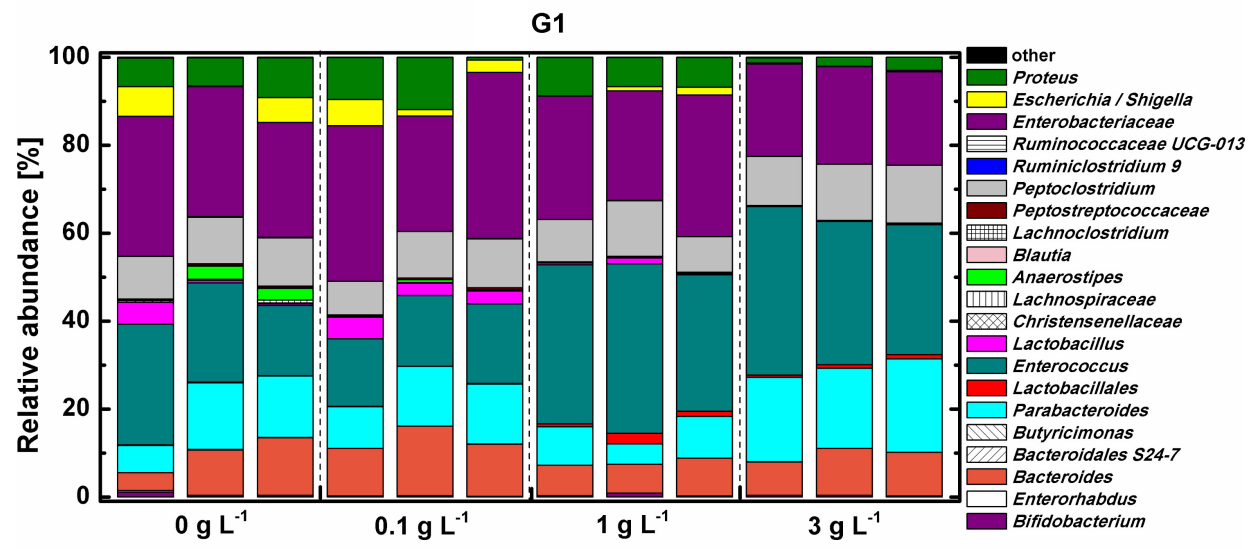

B

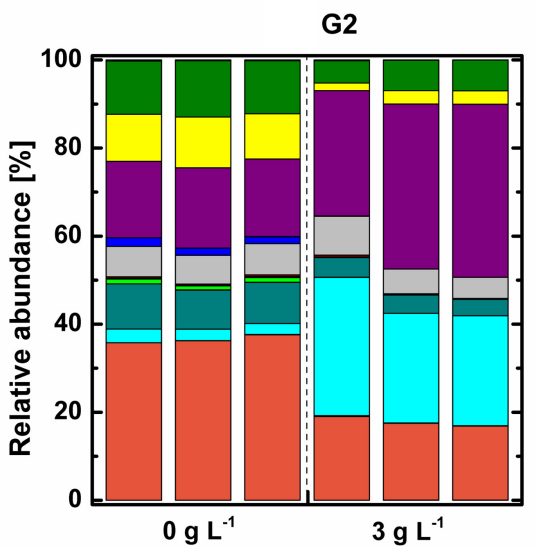

C

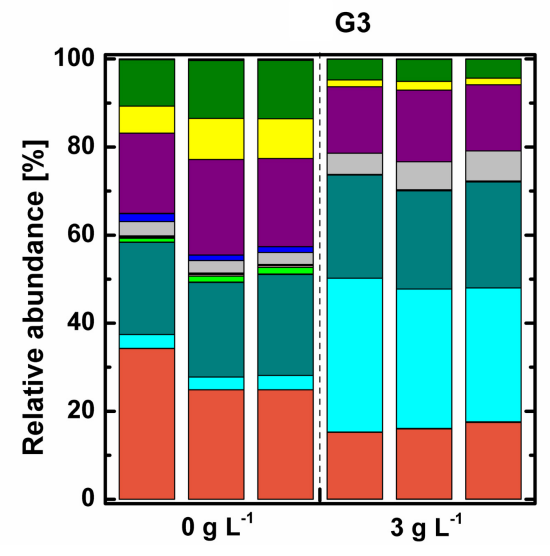

FIGURE 4 | Relative abundance of bacterial community in mice fecal samples at genus level. Mice fecal samples treated with $0,0.1,1,3 \mathrm{~g} \mathrm{~L}^{-1} \mathrm{COS}$ at $72 \mathrm{~h}$ of the primary culture (G1) (A), 0 or $3 \mathrm{~g} \mathrm{~L}^{-1} \mathrm{COS}$ at the end of the 2 nd subculture (G2) (B), and 0 or $3 \mathrm{~g} \mathrm{~L}^{-1} \mathrm{COS}$ at the end of the 3rd subculture (G3) (C).

COS, the abundance of the phylum Verrucomicrobia was significantly increased $(P<0.05)$, while Firmicutes and Proteobacteria was remarkably decreased $(P<0.05)$. Moreover, the abundance of the phylum Bacteroidetes in the COS group showed a trend of increase, however, it was not significantly different from that in the control. At genus level (Figures 5B,C), there were several bacteria increased in the COS group $(P<0.05)$, including Coriobacteriaceae, Allobaculum, Turicibacter, Akkermansia, and Peptococcaceae. On the contrary, the abundances of Lactobacillus, Desulfovibrio, Bifidobacterium, Mucispirillum, vadinBB60, Acetatifactor and Intestinimonas were dramatically suppressed with the administration of COS $(P<0.05$ or $P<0.01)$. In addition, the relative abundance of Mucispirillum, vadinBB60 and Acetatifactor were below $0.5 \%$ in the COS group.

\section{DISCUSSION}

Gut microbiota is associated with not only food digestion and metabolism, but also gut heath and several diseases
(Clemente et al., 2012). COS have shown versatile healthrelated biological functions such as immune-stimulation, antimicrobial activity (Liaqat and Eltem, 2018). Those biological functions might be related to interaction with gut microbiota. An important result of our present studies is a comprehensive and comparative analysis of the microbial community from mice fecal samples treated with different concentrations of COS both in vitro and in animal model. Addition of $1 \mathrm{~g} \mathrm{~L}^{-1}$ or higher concentration of COS significantly inhibited the growth of fecal bacteria in vitro. However, following the elongation of the incubation time, the total number of bacteria was quickly returned to the same level of bacteria in the untreated control (Table 1), likely due to the consumption of COS by the microbe (Figures 1, 2). Interestingly, the utilization rate was different among COS oligomers, in which COS-5 was obviously not favored by the fecal microbe. At the phylum level, the abundance of Bacteroidetes was the significantly increased, while Proteobacteria was remarkably decreased under $3 \mathrm{~g} \mathrm{~L}^{-1}$ COS treatment at the end of the 3rd subculture. Moreover, amounts of genera Bacteroides, Proteus, and Escherichia/Shigella, were significantly decreased and Parabacteroides spp. was increased 
A

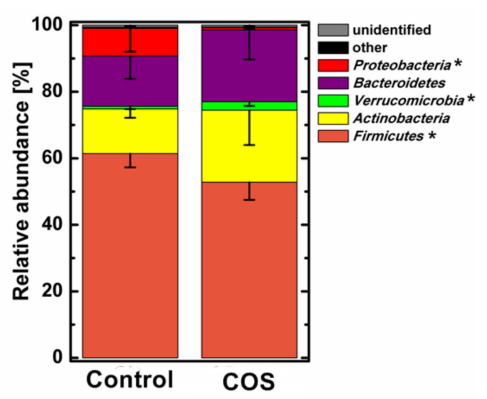

C

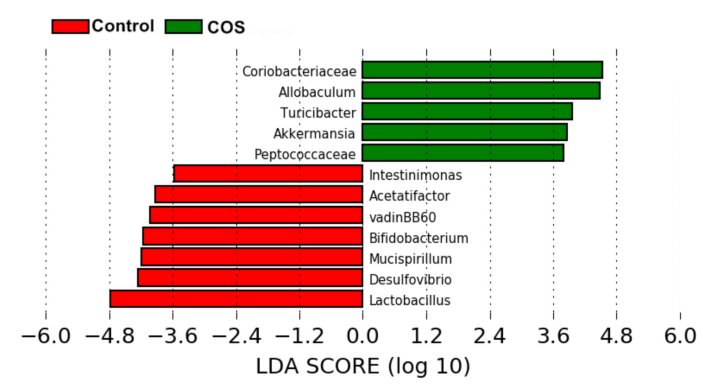

B

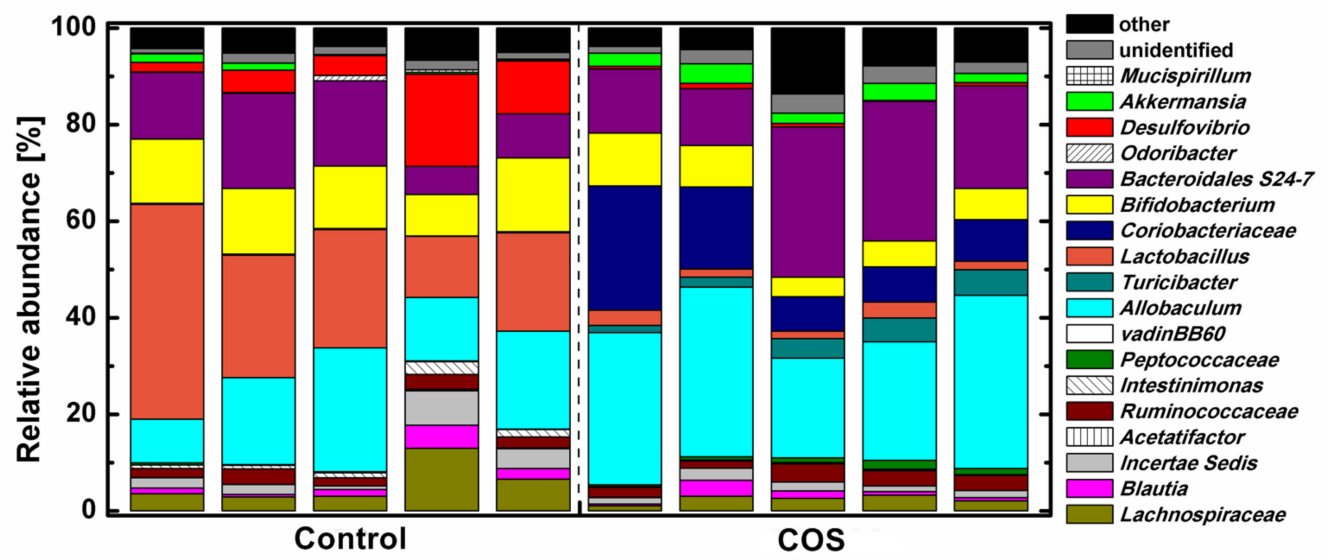

FIGURE $\mathbf{5}$ | Effect of COS treatment on the structure of gut bacterial community in mice. Relative abundance of bacterial community at phylum level (A) genus level (B). Significant differences of the bacteria taxa in mice with or without COS treatments based on Linear discriminant analysis effect size (LEfSe) (C). Significant differences in LDA scores $(P<0.05)$ were produced among classes (Kruskal-Wallis test) and between subclasses (Wilcoxon's test). The threshold logarithmic LDA score was 3.6. Significant differences were noted as follows: ${ }^{P} P<0.05$.

under COS treatments in vitro. The trend was reinforced by increased concentration of COS and times of subculture. Changes on the production of several kinds of SCFA from in vitro fermentation were also identified. The relationship among COS, microbe and metabolites was also preliminarily established. In the mouse model, the abundance of phylum Firmicutes and Proteobacteria was remarkably decreased in the colon contents of mice fed with COS. At the genus level, supplementation of COS obviously increased the abundance of beneficial bacteria Akkermansia, and greatly decreased the relative abundance of harmful bacteria Desulfovibrio (phylum Proteobacteria).

Chitosan oligosaccharides have been shown to exhibit inhibitory effects against both Gram-positive and Gram-negative bacteria with the minimum inhibitory concentration (MIC) of 0.8-1.2 $\mathrm{g} \mathrm{L}^{-1}$ (Kittur et al., 2005; Wang et al., 2007). Our studies also found that copy numbers of bacterial 16S rRNA genes in samples treated with $0.1,1$, and $3 \mathrm{~g} \mathrm{~L}^{-1}$ COS were decreased 2.7, 300, 1200 times compared to the control, respectively. However, there was no significant difference in 16S rRNA genes copy numbers between the COS treated groups and the control after incubation for $72 \mathrm{~h}$. Same trend was observed
TABLE 1 | Abundance of bacterial population through COS treatment.

\begin{tabular}{|c|c|c|c|}
\hline \multirow{2}{*}{$\begin{array}{l}\text { COS Concentration } \\
{\left[\mathrm{g} \mathrm{L}^{-1}\right]}\end{array}$} & \multicolumn{3}{|c|}{$\log _{10}$ [Bacteria 16S rRNA gene (copies $\mathrm{mL}^{-1}$ )] } \\
\hline & Inocula & $8 \mathrm{~h}$ & $72 \mathrm{~h}$ \\
\hline 0 & $11.32 \pm 0.07$ & $11.54 \pm 0.08^{\mathrm{a}}$ & $12.30 \pm 0.08^{a b}$ \\
\hline 0.1 & & $11.10 \pm 0.1^{b}$ & $12.35 \pm 0.08^{a}$ \\
\hline 1 & & $9.02 \pm 0.1^{\mathrm{c}}$ & $12.27 \pm 0.07^{\mathrm{ab}}$ \\
\hline 3 & & $8.44 \pm 0.1^{d}$ & $12.12 \pm 0.06^{b}$ \\
\hline
\end{tabular}

Abundance of bacteria was estimated by quantitative PCR analyses based on bacteria $16 S$ rRNA gene copy numbers in the liquid medium containing mice feces treated with $0,0.1,1$, or $3 \mathrm{~g} \mathrm{~L}^{-1}$ COS, respectively. Data were collected at 0 , 8 , and $72 \mathrm{~h}$ of the treatment. Data are represented as the means $\pm S D(n=3)$. Superscripts with different letters indicate significant differences $(P<0.05)$ between treatments at the same time point.

when Lactobacillus spp. strains were treated with COS in pure cultures (Supplementary Figure S8). Thus, the inhibitory effects of COS on bacteria declined in accordance with the extension of the treatment time. Consistently, the concentration of COS in the sample dropped down quickly (Figure 1), indicating the consumption of COS by the microbe. In addition, cellulose as a 
limited fermentability control is helpful for confirming COS as microbiota-accessible carbohydrates.

Degree of polymerization of COS is tightly associated with its bio-activities (Kittur et al., 2005; Mateos-Aparicio et al., 2016). In the present study, COS oligomers showed different degrading dynamics by the fecal microbe: slower degradation of COS with higher DP (Figure 1). The transient accumulation of COS with lower DP might be due to the degradation of larger oligomers. Interestingly, degradation of COS-5 was obviously slower than others, suggesting the utilization of COS-5 was relatively difficult or unfavorable for the microbe (Figure 1E). In fact both direct cellular uptake and extracellular enzymatic degradation might contribute to COS consumption by gut microbe. Studies showed that gut bacteria was able to directly uptake a variety of oligosaccharides through specific transporters (Abou Hachem et al., 2013). A recent study showed that cell uptake of COS dimer (COS-2) was faster than trimer (COS-3) by S. coelicolor (Viens et al., 2015), suggesting lower DP was more favorable for the bacteria. Furthermore, chitosanase have been found in a variety of microorganisms, and was usually expressed extracellularly (Jo et al., 2003; Liu et al., 2015). Interestingly, studies on GH8 and GH46 chitosanase families indicated that the substrates associated with them were both composed of six subsites (Liu et al., 2015). Moreover, the rate of COS degradation by some chitosanase obviously decreased with a decrease in the substrate size, with a 20 -fold difference on the degradation rate between COS- 6 and COS-5 (Jo et al., 2003). Thus, COS-5 might be unfavorable for both extracellular chitosanase degradation and cellular uptake of gut microbe. This observation brought an important proof for the structural and functional specificity of each COS oligomer. Thus, to better understand the structuralfunctional relationship of COS, purified COS oligomers should be used in the future studies.

Genera Bacteroides and Proteus and Escherichia/Shigella were significantly inhibited by COS treatment (Supplementary Figure S6). The inhibitory effect was enhanced with increased concentration of COS. Metagenomic studies had noted associations between certain species of Bacteroides and diabetes (Johnson et al., 2017). Qin et al. (2012) observed the genus Bacteroides was more abundant in type II diabetic cohorts and Karlsson et al. (2013) got the similar results. The Proteus spp. bacteria were mostly known as opportunistic human pathogens associated with complicated urinary tract and wound infections as well as nosocomial infections (Armbruster and Mobley, 2012). Escherichia/Shigella were leading pathogens, which could cause diarrhea worldwide. On the other hand, the relative abundance of Parabacteroides spp. was significantly increased under $3 \mathrm{~g}$ $\mathrm{L}^{-1}$ COS treatments (Supplementary Figure S6). Members of the phylum Bacteroidetes, including genus Parabacteroides, participated in provisioning the host with energy harvested through the fermentation of indigestible polysaccharides to produce SCFAs (Johnson et al., 2017), and were also related to butyrate production (Palakawong $\mathrm{Na}$ Ayudthaya et al., 2018). Moreover, colonic hydrogen concentration in rats was positively correlated with the abundance of genus Parabacteroides (Nishimura et al., 2018). Those results indicated that Parabacteroides spp. might contribute to the increased concentration of hydrogen and SCFAs through COS treatments (Figures 1-3). Butyrate was an important substrates in the degradation of non-digestible carbohydrates, rather than by protein fermentation under anaerobic conditions (Louis and Flint, 2017). During the anaerobic incubation, we did not detect the production of methane by GC analysis. In addition, there was no electron acceptors, such as nitrate or sulfate in the culture medium. Therefore, butyrate significantly accumulated in the in vitro incubation system. In the host in vivo environment, SCFAs reaching total concentrations of $50-200 \mathrm{mM}$ in the human large intestine could be taken up efficiently by the gut mucosa and have important positive impacts upon host physiology (Koh et al., 2016; Louis and Flint, 2017). Especially, butyrate could be used preferentially as an energy source by the gut mucosa and most often considered to benefit health, including protection against colorectal cancer (Koh et al., 2016; Morrison and Preston, 2016). Thus, these results suggested that COS could positively manipulate the composition of microbial community and stimulate the production of SCFAs, which might bring benefits to gut health.

In animal study, COS as a potential non-digestable oligosaccharide for the host, could be metabolized by gut microbiota. Supplementation of COS greatly decreased the abundance of phylum Proteobacteria (Supplementary Figure S5), consistent with the result of in vitro study. The relative abundance of genus Desulfovibrio (phylum Proteobacteria), which may contribute to colorectal cancer development (Carbonero et al., 2012), greatly decreased with the supplementation of COS (Figure 5). Supplementation of COS also significantly inhibited probiotic genus Lactobacillus (Figure 5), consistent with the result of in vitro study. The probiotic genus Bifidobacterium was also significant inhibited in mice fed with COS. These results were consistent with previous observations (Mateos-Aparicio et al., 2016), and might indicate that COS should not be considered as traditional prebiotic. However, COS greatly elevated the abundance of beneficial genus Akkermansia in mice gut (Figure 5). Genus Akkermansia was involved in the enterocytes proliferation of the colonic wounds and reversing high-fat diet induced metabolic disorders, including fat-mass gain, adipose tissue inflammation and insulin resistance (Everard et al., 2013; Alam et al., 2016). It has to be noted that those beneficial effects of bacteria Akkermansia were in accordance with the biological activities of COS (Liaqat and Eltem, 2018). With the establishment of the exact role of intestinal microbial flora and the increasing species of probiotics, the scope and definition of prebiotics would be expanded and revised in the future (Bindels et al., 2015). Based on the beneficial effects of COS on host, it might be considered to be a kind of potential prebiotics. In addition, supplementation of COS increased the abundance of Coriobacteriaceae, which exhibited importance functions, such as the conversion of bile salts and steroids as well as the activation of dietary polyphenols (Clavel et al., 2006; Ridlon et al., 2006). Phylum Proteobacteria was significantly inhibited by COS both in the animal model and in vitro fermentation. However, at the genus level, structures of bacterial community were relative different between the animal model and 
in vitro fermentation. The difference between in vivo and in vitro environments led different effects of COS on gut microbiota.

Thus, COS might positively reform the composition of microbial community in both in vitro fermentation and animal studies, although affected bacteria taxa were difference between two experimental conditions. These results indicated the potential beneficial effects of COS on the host health through reforming the structure of intestinal bacteria.

\section{CONCLUSION}

We reported the effects of COS on intestinal bacteria of mice in vivo and in vitro. During in vitro fecal cultivation, COS significantly inhibited the growth and diversity of total bacteria community at the beginning of incubation. The inhibitory effect of COS obviously declined with the utilization of COS by fecal microbe. The abundance of Bacteroides and Escherichia/Shigella decreased with the COS addition. However, COS could increase the abundance of Parabacteroides. In animal study, COS reduced the abundance of probiotic Lactobacillus, Bifidobacterium and harmful bacteria Desulfovibrio, and increased abundance of Akkermansia, which was in accordance with the beneficial activities of COS on host health. Phylum Proteobacteria was significantly inhibited by COS both in the animal model and in vitro fermentation. These results suggested that COS could result in substantial reform of the intestinal microbial community, which might contribute to the health status of the host.

\section{REFERENCES}

Abou Hachem, M., Andersen, J. M., Barrangou, R., Moller, M. S., Fredslund, F., Majumder, A., et al. (2013). Recent insight into oligosaccharide uptake and metabolism in probiotic bacteria. Biocatal. Biotransformation 31, 226-235. doi: 10.3109/10242422.2013.828048

Alam, A., Leoni, G., Quiros, M., Wu, H., Desai, C., Nishio, H., et al. (2016). The microenvironment of injured murine gut elicits a local pro-restitutive microbiota. Nat. Microbiol. 1:15021. doi: 10.1038/nmicrobiol.2015.21

Armbruster, C. E., and Mobley, H. L. (2012). Merging mythology and morphology: the multifaceted lifestyle of Proteus mirabilis. Nat. Rev. Microbiol. 10, 743-754. doi: $10.1038 /$ nrmicro2890

Berger, J., Reist, M., Mayer, J. M., Felt, O., and Gurny, R. (2004). Structure and interactions in chitosan hydrogels formed by complexation or aggregation for biomedical applications. Eur. J. Pharm. Biopharm. 57, 35-52. doi: 10.1016/ S0939-6411(03)00160-7

Bindels, L. B., Delzenne, N. M., Cani, P. D., and Walter, J. (2015). Towards a more comprehensive concept for prebiotics. Nat. Rev. Gastroenterol. Hepatol. 12, 303-310. doi: 10.1038/nrgastro.2015.47

Cantarel, B. L., Lombard, V., and Henrissat, B. (2012). Complex carbohydrate utilization by the healthy human microbiome. PLoS One 7:e28742. doi: 10.1371/ journal.pone.0028742

Caporaso, J. G., Kuczynski, J., Stombaugh, J., Bittinger, K., Bushman, F. D., Costello, E. K., et al. (2010). QIIME allows analysis of high-throughput community sequencing data. Nat. Methods 7, 335-336. doi: 10.1038/nmeth.f. 303

Carbonero, F., Benefiel, A. C., Alizadeh-Ghamsari, A. H., and Gaskins, H. R. (2012). Microbial pathways in colonic sulfur metabolism and links with health and disease. Front. Physiol. 3:448. doi: 10.3389/fphys.2012.00448

\section{AUTHOR CONTRIBUTIONS}

$\mathrm{CZ}$ and YD participated in the design of the study. CZ conducted the experiments and analyzed the data. SJ analyzed the volatile fatty acids and chitosan oligosaccharides. CZ wrote the first draft of the manuscript. ZW revised the manuscript. All authors read and approved the final manuscript.

\section{FUNDING}

This study was financially supported by the National Natural Science Foundation of China (41501251 and U1608255) and the Key Program of the Chinese Academy of Sciences (KFZD-SW218-1).

\section{ACKNOWLEDGMENTS}

We thank Dr. Ke Ma (College of Resources and Environmental Sciences, China Agricultural University) for the help in gas analysis and Junping Zheng, Hongtao Liu (Institute of Process Engineering, Chinese Academy of Sciences) for the help in feeding mice.

\section{SUPPLEMENTARY MATERIAL}

The Supplementary Material for this article can be found online at: https://www.frontiersin.org/articles/10.3389/fmicb. 2018.02388/full\#supplementary-material

Choi, B. K., Kim, K. Y., Yoo, Y. J., Oh, S. J., Choi, J. H., and Kim, C. Y. (2001). In vitro antimicrobial activity of a chitooligosaccharide mixture against Actinobacillus actinomycetemcomitans and Streptococcus mutans. Int. J. Antimicrob. Agents 18, 553-557. doi: 10.1016/S0924-8579(01)00 434-4

Clavel, T., Dore, J., and Blaut, M. (2006). Bioavailability of lignans in human subjects. Nutr. Res. Rev. 19, 187-196. doi: 10.1017/S0954422407249704

Clemente, J. C., Ursell, L. K., Parfrey, L. W., and Knight, R. (2012). The impact of the gut microbiota on human health: an integrative view. Cell 148, 1258-1270. doi: 10.1016/j.cell.2012.01.035

Du, Y. Z., Wang, L., Yuan, H., Wei, X. H., and Hu, F. Q. (2009). Preparation and characteristics of linoleic acid-grafted chitosan oligosaccharide micelles as a carrier for doxorubicin. Colloids Surf. B Biointerfaces 69, 257-263. doi: 10.1016/j.colsurfb.2008.11.030

El Kaoutari, A., Armougom, F., Gordon, J. I., Raoult, D., and Henrissat, B. (2013). The abundance and variety of carbohydrate-active enzymes in the human gut microbiota. Nat. Rev. Microbiol. 11, 497-504. doi: 10.1038/nrmicro 3050

Everard, A., Belzer, C., Geurts, L., Ouwerkerk, J. P., Druart, C., Bindels, L. B., et al. (2013). Cross-talk between Akkermansia muciniphila and intestinal epithelium controls diet-induced obesity. Proc. Natl. Acad. Sci. U.S.A. 110, 9066-9071. doi: 10.1073/pnas. 1219451110

Goodman, A. L., Kallstrom, G., Faith, J. J., Reyes, A., Moore, A., Dantas, G., et al. (2011). Extensive personal human gut microbiota culture collections characterized and manipulated in gnotobiotic mice. Proc. Natl. Acad. Sci. U.S.A. 108, 6252-6257. doi: 10.1073/pnas.1102938108

Jo, Y. Y., Jo, K. J., Jin, Y. L., Kim, K. Y., Shim, J. H., Kim, Y. W., et al. (2003). Characterization and kinetics of $45 \mathrm{kDa}$ chitosanase from Bacillus sp. p16. Biosci. Biotechnol. Biochem. 67, 1875-1882. doi: 10.1271/bbb.67.1875 
Johnson, E. L., Heaver, S. L., Walters, W. A., and Ley, R. E. (2017). Microbiome and metabolic disease: revisiting the bacterial phylum Bacteroidetes. J. Mol. Med. 95, 1-8. doi: 10.1007/s00109-016-1492-2

Karlsson, F. H., Tremaroli, V., Nookaew, I., Bergstrom, G., Behre, C. J., Fagerberg, B., et al. (2013). Gut metagenome in European women with normal, impaired and diabetic glucose control. Nature 498, 99-103. doi: 10.1038/ nature 12198

Kittur, F. S., Kumar, A. B. V., Varadaraj, M. C., and Tharanathan, R. N. (2005). Chitooligosaccharides-preparation with the aid of pectinase isozyme from Aspergillus niger and their antibacterial activity. Carbohydr. Res. 340, 1239-1245. doi: 10.1016/j.carres.2005.02.005

Koh, A., De Vadder, F., Kovatcheva-Datchary, P., and Backhed, F. (2016). From dietary fiber to host physiology: short-chain fatty acids as key bacterial metabolites. Cell 165, 1332-1345. doi: 10.1016/j.cell.2016.05.041

Lee, H. W., Park, Y. S., Choi, J. W., Yi, S. Y., and Shin, W. S. (2003). Antidiabetic effects of chitosan oligosaccharides in neonatal streptozotocininduced noninsulin-dependent diabetes mellitus in rats. Biol. Pharm. Bull. 26, 1100-1103. doi: 10.1248/bpb. 26.1100

Lee, H. W., Park, Y. S., Jung, J. S., and Shin, W. S. (2002). Chitosan oligosaccharides, dp 2-8, have prebiotic effect on the Bifidobacterium bifidium and Lactobacillus sp. Anaerobe 8, 319-324. doi: 10.1016/S1075-9964(03)00030-1

Liaqat, F., and Eltem, R. (2018). Chitooligosaccharides and their biological activities: a comprehensive review. Carbohydr. Polym. 184, 243-259. doi: 10.1016/j.carbpol.2017.12.067

Liu, S. J., Shao, S. J., Li, L. L., Cheng, Z., Tian, L., Gao, P. J., et al. (2015). Substrate-binding specificity of chitinase and chitosanase as revealed by activesite architecture analysis. Carbohydr. Res. 418, 50-56. doi: 10.1016/j.carres.2015. 10.002

Louis, P., and Flint, H. J. (2017). Formation of propionate and butyrate by the human colonic microbiota. Environ. Microbiol. 19, 29-41. doi: 10.1111/14622920.13589

Lueders, T., Manefield, M., and Friedrich, M. W. (2004). Enhanced sensitivity of DNA- and rRNA-based stable isotope probing by fractionation and quantitative analysis of isopycnic centrifugation gradients. Environ. Microbiol. 6, 73-78. doi: 10.1046/j.1462-2920.2003.00536.x

Mateos-Aparicio, I., Mengibar, M., and Heras, A. (2016). Effect of chitooligosaccharides over human faecal microbiota during fermentation in batch cultures. Proc. Natl. Acad. Sci. U.S.A. 137, 617-624. doi: 10.1016/j.carbpol.2015. 11.011

Morrison, D. J., and Preston, T. (2016). Formation of short chain fatty acids by the gut microbiota and their impact on human metabolism. Gut Microbes 7, 189-200. doi: 10.1080/19490976.2015.1134082

Muanprasat, C., and Chatsudthipong, V. (2017). Chitosan oligosaccharide: biological activities and potential therapeutic applications. Pharmacol. Ther. 170, 80-97. doi: 10.1016/j.pharmthera.2016.10.013

Nishimura, N., Tanabe, H., Komori, E., Sasaki, Y., Inoue, R., and Yamamoto, T. (2018). Transplantation of high hydrogen-producing microbiota leads to generation of large amounts of colonic hydrogen in recipient rats fed high amylose maize starch. Nutrients 10:E144. doi: 10.3390/nu10020144

Palakawong Na Ayudthaya, S., van de Weijer, A. H. P., van Gelder, A. H., Stams, A. J. M., de Vos, W. M., and Plugge, C. M. (2018). Organic acid production from potato starch waste fermentation by rumen microbial communities from Dutch and Thai dairy cows. Biotechnol. Biofuels 11:13. doi: 10.1186/s13068-018-10124

Payne, A. N., Zihler, A., Chassard, C., and Lacroix, C. (2012). Advances and perspectives in in vitro human gut fermentation modeling. Trends Biotechnol. 30, 17-25. doi: 10.1016/j.tibtech.2011.06.011

Qin, J., Li, Y., Cai, Z., Li, S., Zhu, J., Zhang, F., et al. (2012). A metagenomewide association study of gut microbiota in type 2 diabetes. Nature 490, 55-60. doi: $10.1038 /$ nature 11450
Rastall, R. A. (2010). Functional oligosaccharides: application and manufacture. Annu. Rev. Food Sci. Technol. 1, 305-339. doi: 10.1146/annurev.food.080708. 100746

Ridlon, J. M., Kang, D. J., and Hylemon, P. B. (2006). Bile salt biotransformations by human intestinal bacteria. J. Lipid Res. 47, 241-259. doi: 10.1194/jlr. R500013-JLR200

Segata, N., Izard, J., Waldron, L., Gevers, D., Miropolsky, L., Garrett, W. S., et al. (2011). Metagenomic biomarker discovery and explanation. Genome Biol. 12:R60. doi: 10.1186/gb-2011-12-6-r60

Shang, W., Si, X., Zhou, Z., Li, Y., Strappe, P., and Blanchard, C. (2017). Characterization of fecal fat composition and gut derived fecal microbiota in high-fat diet fed rats following intervention with chito-oligosaccharide and resistant starch complexes. Food Funct. 8, 4374-4383. doi: 10.1039/c7fo01244f

Thadathil, N., and Velappan, S. P. (2014). Recent developments in chitosanase research and its biotechnological applications: a review. Food Chem. 150, 392-399. doi: 10.1016/j.foodchem.2013.10.083

Vernazza, C. L., Gibson, G. R., and Rastall, R. A. (2005). In vitro fermentation of chitosan derivatives by mixed cultures of human faecal bacteria. Carbohydr. Polym. 60, 539-545. doi: 10.1016/j.carbpol.2005.03.008

Viens, P., Dubeau, M. P., Kimura, A., Desaki, Y., Shinya, T., Shibuya, N., et al. (2015). Uptake of chitosan-derived D-glucosamine oligosaccharides in Streptomyces coelicolor A3(2). FEMS Microbiol. Lett. 362:fnv048. doi: 10.1093/ femsle/fnv048

Wang, Y., Zhou, P. G., Yu, J. X., Pan, X. R., Wang, P. P., Lan, W. Q., et al. (2007). Antimicrobial effect of chitooligosaccharides produced by chitosanase from Pseudomonas CUY8. Asia Pac. J. Clin. Nutr. 16, 174-177.

Yang, C. M., Ferket, P. R., Hong, Q. H., Zhou, J., Cao, G. T., Zhou, L., et al. (2012). Effect of chito-oligosaccharide on growth performance, intestinal barrier function, intestinal morphology and cecal microflora in weaned pigs. J. Anim. Sci. 90, 2671-2676. doi: 10.2527/jas.2011-4699

Yousef, M., Pichyangkura, R., Soodvilai, S., Chatsudthipong, V., and Muanprasat, C. (2012). Chitosan oligosaccharide as potential therapy of inflammatory bowel disease: therapeutic efficacy and possible mechanisms of action. Pharmacol. Res. 66, 66-79. doi: 10.1016/j.phrs.2012.03.013

Yu, T., Wang, Y., Chen, S., Hu, M., Wang, Z., Wu, G., et al. (2017). Low-molecularweight chitosan supplementation increases the population of Prevotella in the cecal contents of weanling pigs. Front. Microbiol. 8:2182. doi: 10.3389/fmicb. 2017.02182

Zhang, C., Yuan, Q., and Lu, Y. (2014). Inhibitory effects of ammonia on methanogen mcrA transcripts in anaerobic digester sludge. FEMS Microbiol. Ecol. 87, 368-377. doi: 10.1111/1574-6941.12229

Zhang, H., Du, Y., Yu, X., Mitsutomi, M., and Aiba, S. (1999). Preparation of chitooligosaccharides from chitosan by a complex enzyme. Carbohydr. Res. 320, 257-260. doi: 10.1016/S0008-6215(99)00154-8

Zou, P., Yang, X., Wang, J., Li, Y., Yu, H., Zhang, Y., et al. (2016). Advances in characterisation and biological activities of chitosan and chitosan oligosaccharides. Food Chem. 190, 1174-1181. doi: 10.1016/j.foodchem.2015. 06.076

Conflict of Interest Statement: The authors declare that the research was conducted in the absence of any commercial or financial relationships that could be construed as a potential conflict of interest.

Copyright (c) 2018 Zhang, Jiao, Wang and Du. This is an open-access article distributed under the terms of the Creative Commons Attribution License (CC BY). The use, distribution or reproduction in other forums is permitted, provided the original author(s) and the copyright owner(s) are credited and that the original publication in this journal is cited, in accordance with accepted academic practice. No use, distribution or reproduction is permitted which does not comply with these terms. 